\title{
Lessons from the First Year of the International Bio-Logging Society's Data Standardisation Working Group
}

\author{
Peggy Newman ${ }^{\ddagger}$, Francesca Cagnacci ${ }^{\S}$, Sarah Davidson ${ }^{1, \pi}$ \\ ‡ Atlas of Living Australia, Canberra, Australia \\ $\S$ Fondazione Edmund Mach IASMA, Trento, Italy \\ | Max Planck Institute for Ornithology, Radolfzell, Germany \\ I The Ohio State University, Columbus, United States of America
}

Corresponding author: Peggy Newman (peggydnewman@gmail.com), Francesca Cagnacci (francesca.cagnacci@f mach.it), Sarah Davidson (sdavidson@orn.mpg.de)

Received: 08 Aug 2019 | Published: 20 Aug 2019

Citation: Newman P, Cagnacci F, Davidson S (2019) Lessons from the First Year of the International Bio-Logging Society's Data Standardisation Working Group. Biodiversity Information Science and Standards 3: e38919.

https://doi.org/10.3897/biss.3.38919

\section{Abstract}

The Data Standardisation Working Group pursues the recently formed International BioLogging Society's (IBioLS) objective "to progress standardisation of data protocols used within the bio-logging community, with a view to making databases interoperable". During 2017 and 2018, the group has garnered a lot of interest across the sector from well over 200 colleagues with broad international representation from device manufacturers, researchers, biodiversity data experts and bio-logging database managers.

Through a series of remote meetings, the group has explored a range of existing, relevant standards, projects and platforms that could be leveraged to facilitate data decoding, exchange, archiving and discoverability.

This presentation will highlight some of the research and examples discussed by the group including the Open Geospatial Consortium (OGC) and W3C sensor-based standards that are being adopted in similar sectors, Darwin Core and the OBIS-ENV-DATA Darwin Core format as a way to define completed datasets, NASA's Oceanographic In-situ data 
Interoperability Project ( $\mathrm{OIIP}$ ), which developed prototype templates for biologging data, and the NERC Vocabulary Server for managing persistent terms.

Considerable challenges ahead lie in resourcing the development of standards, enabling technical leadership, and negotiating governance and consensus in a domain where most of the stakeholders participate in a common market as either manufacturers or consumers of sensor infrastructure.

\section{Presenting author}

Peggy Newman

\section{Presented at}

Biodiversity_Next 2019 